\title{
Brecha de género en la autoría de artículos publicados en el Boletín Médico del Hospital Infantil de México
}

\author{
Karla P. Estrada-Ramírez ${ }^{1}$, América L. Miranda-Lora ${ }^{2}$, Ricardo C. Sandoval-Quiroa ${ }^{3}$, Diana Ávila-Montiel${ }^{4}$, \\ María J. Mier-Prado 5 y Juan Garduño-Espinosa ${ }^{4 *}$ \\ ${ }^{1}$ Departamento de Medicina Interna; ${ }^{2}$ Unidad de Investigación en Medicina Basada en Evidencias; ${ }^{3}$ Departamento de Servicio Social; ${ }^{4}$ Dirección \\ de Investigación; ${ }^{5}$ Departamento de Pediatría. Hospital Infantil de México Federico Gómez, Ciudad de México, México
}

\begin{abstract}
Resumen
Introducción: La participación de las mujeres en medicina ha incrementado en las últimas décadas, incluyendo mayor representatividad en la autoría de artículos científicos en diversos países y distintas especialidades. El objetivo del estudio fue analizar las diferencias de género en la autoría de artículos a través de la historia del Boletín Médico del Hospital Infantil de México. Métodos: Se realizó un análisis bibliométrico de los artículos originales publicados en los años 1953, 1963, 1973, 1983, 1993, 2003 y 2013. Se identificó el género de los autores, tipo de autoría (primer autor o autor correspondiente) y el diseño de estudio (descriptivo vs. analítico). Se evaluó la diferencia en la proporción de género y la tendencia en el tiempo. Resultados: Se incluyeron 272 artículos. Se observó una reducción en la brecha de género entre 1953 y 2013. La participación de las mujeres como primeras autoras incrementó del 2 al $63 \%(p<0.001)$ y como correspondiente del 27 al $59 \%(p<0.001)$. Al considerar únicamente estudios analíticos, el incremento fue del 25 al $50 \%$ como primera autora ( $p=0.03)$, con un cambio similar como autor correspondiente, pero sin una tendencia significativa en el tiempo $(p=0.19)$. La reducción en la brecha de género fue más notoria a partir del periodo 1983-1993. Conclusiones: En las últimas décadas ha existido un incremento significativo en la autoría de las mujeres en el Boletín Médico del Hospital Infantil de México, llegando incluso a una mayor proporción en relación con el sexo masculino. Lo anterior es un reflejo del papel actual de las mujeres en la medicina; en particular, en el área pediátrica.
\end{abstract}

Palabras clave: Brecha de género. Pediatría. Mujeres.

\section{Gender gap in the authorship of published articles in the Boletín Médico del Hospital Infantil de México}

\section{Abstract}

Background: Women's participation in medicine has increased in the last decades, with greater representativeness in the authorship of scientific articles in many countries and different specialties. The objective of this research was to analyze the gender gap in the authorship of articles through the history of the medical journal Boletín Médico del Hospital Infantil de México. Methods: In a bibliometric analysis, we reviewed original articles published during the years 1953, 1963, 1973, 1983, 1993, 2003 and 2013. The gender of the author, type of authorship (first author or corresponding author) and the design of the study (descriptive vs analytic) were identified. We evaluated the difference between gender proportion and trends over

Correspondencia:

*Juan Garduño-Espinosa

E-mail: juan.gardunoe@gmail.com
Disponible en internet: 10-08-2018 Bol Med Hosp Infant Mex. 2018;75:216-223 www.bmhim.com

1665-1146/@ 2018. Hospital Infantil de México Federico Gómez, impreso por Permanyer México SA de CV, todos los derechos reservados. 
time. Results: We included 272 articles. We observed a gender gap reduction between 1953 and 2013. The participation of women as first author increased from 2 to $63 \%(p<0.001)$ and as corresponding author from 27 to $59 \%(p<0.001)$. If we include only analytic studies, the increasing was 25 to $50 \%$ as first author $(p=0.03)$, with a similar tendency as corresponding author, but without a statistical significant on time $(p=0.19)$. We observed the most notable change since 1983-1993. Conclusions: In the last decades, there has been a significant increase in women's authorship in the medical journal Boletín Médico del Hospital Infantil de México, even reaching a greater proportion against male gender. This reflects the present role of women in medicine, particularly in pediatrics.

Key words: Gender gap. Pediatrics. Women.

\section{Introducción}

A lo largo del tiempo, los términos sexo y género han evolucionado según la época o sociedad, y con frecuencia se confunden debido a su naturaleza.

El término sexo se refiere a las características físicas, biológicas y sexuales de las personas. Este término va orientado a la posibilidad de reproducción sexual, lo que clasifica a las personas en hombres y mujeres. Sin embargo, en la actualidad, se incluyen categorías como mujer, hombre, intersexual (personas con características sexuales masculinas y femeninas) y transexual (personas sometidas a cirugías o tratamientos hormonales para cambio de sexo) ${ }^{1}$.

Por otro lado, el término género es una construcción sociocultural que gira en torno a la percepción de lo que corresponde a las ideas, normas y comportamientos que la sociedad espera o establece a lo femenino o masculino; es decir, surge de un sistema de prácticas y relaciones sociales ${ }^{1,2}$.

Las relaciones sociales de género se determinan según el sexo de las personas. Esto ocasiona una relación asimétrica entre hombres y mujeres; es decir, se establece una diferencia entre ambos donde se consideran más importantes las actividades y acciones de lo masculino, lo que confiere mayor poder en la sociedad a los hombres y propicia desigualdad para las mujeres ${ }^{2,3}$.

El papel de las mujeres en ámbitos profesionales y laborales ha ido en aumento. Sin embargo, persisten desigualdades de género en distintas áreas. México ocupa el lugar 71 en equidad de género entre 145 países; ello de acuerdo con el Reporte Global de Brecha de Género 2015 publicado por el Foro Económico Mundial, con una puntuación de $0.70(0=$ inequidad, $1=$ igualdad). La mayor inequidad de nuestro país se reporta en las áreas de participación económica y oportunidades $(\leq 0.67)$, así como en el empoderamiento político $(\leq 0.74)$. Por otra parte, rubros como el nivel educativo, salud y sobrevida han alcanzado cifras de una mayor equidad $(\geq 0.95)^{1}$.
Tradicionalmente, la profesión médica y científica había sido dominada por los hombres. Sin embargo, es indudable que la participación de las mujeres ha ido en aumento en las últimas décadas. A pesar de ello, se ha observado que las mujeres continúan siendo minoría en relación con la autoría de artículos en diversas especialidades médicas ${ }^{2}$.

Ello puede deberse a que las mujeres que están en el gremio se encuentran subordinadas en una estructura vertical. Si bien se ha observado un incremento del sexo femenino en la investigación, la brecha de género sigue siendo elevada 4 . Las publicaciones científicas son una herramienta para la medición de la productividad académica. Se esperaría que el incremento de la participación de las mujeres en las distintas especialidades médicas fuera paralelo a un incremento en la producción científica; sin embargo, no siempre resulta cierto. Algunos reportes señalan que continúa persistiendo una brecha de género en las publicaciones científicas en distintas especialidade ${ }^{2-14}$ mientras que otros reportan una notable reducción ${ }^{7,15-17}$.

A la fecha, no existen datos acerca del panorama actual de la participación de las mujeres en la publicación de artículos en México, y menos en el área pediátrica, que ha sido una de las especialidades donde la participación de las mujeres ha incrementado de forma notoria.

Para estudiar la desigualdad en la investigación en la literatura, se han propuesto dos hipótesis. La primera se conoce como efecto cohorte: atribuye la desigualdad a la incorporación tardía de las mujeres al ámbito profesional. En dicha teoría se cree que la igualdad se logrará de forma natural, conforme sigan incorporándose más mujeres al ámbito científico ${ }^{5,6,18}$.

La segunda hipótesis se denomina techo de cristal y señala a la estructura social patriarcal como causante de las dificultades que enfrentan las mujeres para lograr puestos de rangos superiores, incluso existiendo leyes que fomenten la equidad de género ${ }^{19}$. 
El Hospital Infantil de México Federico Gómez ha estado interesado en la generación y difusión de conocimiento y lo hace a través de su órgano informativo, el Boletín Médico del Hospital Infantil de México (BMHIM). Esta publicación tiene más de 70 años difundiendo conocimientos en el área pediátrica de forma ininterrumpida, tanto en México como en otros países de Latinoamérica. El objetivo del presente estudio fue analizar las diferencias de género en la autoría de artículos durante la historia del BMHIM.

\section{Métodos}

Se realizó un estudio bibliométrico en el que se incluyeron los estudios identificados como artículos originales y editoriales publicados en 1953, 1963, 1973, 1983, 1993, 2003 y 2013 en el BMHIM. También se revisó la conformación del comité editorial en dichos años y su disposición según las divisiones por áreas de especialidad.

Se excluyeron revisiones narrativas, comentarios y cartas al editor. Se identificó el género de los autores, tipo de autoría (primer autor o autor correspondiente) y el diseño del estudio (descriptivo vs. analítico). Cabe mencionar que se incluyeron los artículos editoriales, ya que estos se realizan por encargo del comité editorial. En este sentido, resulta importante conocer cuántos de estos artículos fueron encargados a hombres y cuántos a mujeres.

La identificación del género fue realizada por dos evaluadores independientes, quienes consideraron los siguientes aspectos:

- Nombres relacionados con un solo género. Por ejemplo, Federico corresponde a hombre y Aída corresponde a mujer.

- Identificación del género en el título profesional. Por ejemplo, Dr. o Dra.

- Cuando el género del autor no pudo ser definido en la valoración inicial, porque solamente se tenían las iniciales o el nombre no era relacionado solamente un género (por ejemplo, "Guadalupe"), se realizó una búsqueda exhaustiva en internet en sitios personales, institucionales o publicaciones previas, en el intento de definir el género del autor.

- En algunos casos se consultó con colegas para corroborar el género de los autores. Se excluyeron los artículos en los cuales, a pesar de estas medidas, el género no pudo ser identificado.

El primer autor se identificó como el primer nombre que aparece en la lista de autores y el autor correspondiente se identificó de acuerdo con los datos de contacto para envío de correspondencia de cada artículo. En artículos con un solo autor, este fue identificado tanto como primer autor como autor correspondiente.

En relación con el diseño del estudio, se consideraron como estudios analíticos aquellos en los que se tenía un grupo de comparación (transversal analítico, casos y controles, cohortes y ensayos clínicos); como estudios no analíticos, aquellos en los que únicamente se incluía un análisis descriptivo de los datos (transversal descriptivo, serie de casos).

Los artículos originales fueron caracterizados utilizando estadística descriptiva (frecuencias absolutas y relativas). Se calculó la proporción de género en cada uno de los años evaluados de acuerdo con el tipo de autoría y diseño de estudio. Se realizó la prueba de tendencias de Cochran-Armitage para evaluar la tendencia de la proporción de género en el tiempo. Se utilizó el programa estadístico STATA SE v11.0 y se consideró un nivel de significación estadística con un valor de $p<0.05$. En cuanto a los artículos editoriales, la conformación del comité editorial a través de los años y las subdivisiones por áreas de especialidad, fueron caracterizados por estadística descriptiva para conocer la cantidad de artículos editoriales publicados por género, así como la conformación del comité editorial y sus áreas de especialidad.

\section{Resultados}

Se identificaron 275 artículos de investigación en los volúmenes incluidos, de los cuales se excluyó un artículo por no ser posible identificar a los autores y dos artículos en los cuales no se pudo determinar el género, con un total de 272 artículos en el análisis final (98.9\%).

En la Tabla 1 se presenta el número total de artículos, el número de estudios analíticos y el porcentaje de mujeres como primera autora y autora correspondiente por año de publicación. Estos datos muestran un incremento en la proporción de mujeres con el tiempo del 2 al $63 \%$ como primera autora y del 27 al $59 \%$ como autora correspondiente.

La proporción hombre-mujer en la primera autoría fue de 49:1 en 1953; la proporción fue cambiando hasta llegar a ser equivalente en 2003 y se invirtió en 2013, con una relación 0.6:1. En lo que respecta a la autoría correspondiente, esta relación se invirtió de 2.7:1 en 1953 a 0.7:1 en 2013. Como se observa en la Figura 1, la participación de las mujeres en la autoría se incrementó de forma significativa en el tiempo a partir del periodo 1983-1993 $(p<0.001)$. 
Tabla 1. Número total de artículos, frecuencia de estudios analíticos y porcentaje de autoría de las mujeres por año de publicación

\begin{tabular}{|l|c|c|c|c|c|c|}
\hline Año & $\begin{array}{c}\text { Total de } \\
\text { artículos }\end{array}$ & $\begin{array}{c}\text { Mujeres como } \\
\text { primer autor }\end{array}$ & $\begin{array}{c}\text { Mujeres como autor } \\
\text { correspondiente }\end{array}$ & $\begin{array}{c}\text { Total de artículos } \\
\text { analíticos }\end{array}$ & $\begin{array}{c}\text { Mujeres como } \\
\text { primer autor en } \\
\text { estudios analíticos }\end{array}$ & $\begin{array}{c}\text { Mujeres como autor } \\
\text { correspondiente en } \\
\text { estudios analíticos }\end{array}$ \\
\cline { 2 - 7 } & $\mathbf{n}$ & $\mathbf{n ( \% )}$ & $\mathbf{n ( \% )}$ & $\mathbf{n}$ & $\mathbf{n}(\%)$ & $\mathbf{n}(\%)$ \\
\hline 1953 & 45 & $1(2 \%)$ & $12(27 \%)$ & 4 & $1(25 \%)$ & $1(25 \%)$ \\
\hline 1963 & 32 & $1(3 \%)$ & $4(13 \%)$ & 10 & $1(10 \%)$ & $3(30 \%)$ \\
\hline 1973 & 29 & $4(14 \%)$ & $6(21 \%)$ & 11 & $3(27 \%)$ & $4(36 \%)$ \\
\hline 1983 & 32 & $4(13 \%)$ & $2(6 \%)$ & 19 & $2(11 \%)$ & $1(5 \%)$ \\
\hline 1993 & 66 & $19(29 \%)$ & $14(21 \%)$ & 43 & $12(28 \%)$ & $7(16 \%)$ \\
\hline 2003 & 36 & $18(50 \%)$ & $13(36 \%)$ & 25 & $14(56 \%)$ & $11(44 \%)$ \\
\hline 2013 & 32 & $20(63 \%)$ & $19(59 \%)$ & 22 & $11(50 \%)$ & $11(50 \%)$ \\
\hline Total & 272 & $67(25 \%)$ & $70(26 \%)$ & 134 & $44(33 \%)$ & $38(28 \%)$ \\
\hline
\end{tabular}

Al considerar únicamente estudios analíticos, la proporción hombre-mujer como primer autor se mantuvo en favor del género masculino durante el periodo 19531993, con una relación 2.5-9:1 (Figura 1 C y 1 D). La reducción de la brecha se observó a partir del 2003 y se alcanzó una relación 1:1 en $2013(p=0.031)$. A pesar de que también se observó una inversión de la proporción hombre-mujer como autor correspondiente en los artículos analíticos, estos cambios no mostraron una significación estadística durante el periodo estudiado ( $p=0.195)$.

En cuanto a la conformación del comité editorial correspondiente a los años 1953, 1963, 1973, 1983, 1993, 2003 y 2013, se pudo observar que ninguna mujer formó parte del comité hasta el año 2013. En dicho año, el comité editorial se conformó por el $86 \%$ de hombres y el $14 \%$ de mujeres (Figura 2).

A partir del año 2013, el comité editorial se dividió en ocho áreas de especialidad (biomédica, salud pública, temas pediátricos, educación en salud y ética clínica, casos clínicos, epidemiología clínica, casos clínicos y casos clinicopatológicos). Se pudo observar que en seis de estas áreas (biomédica, temas pediátricos, educación en salud y ética clínica, casos clínicos, epidemiología clínica y casos clinicopatológicos), predominaron los hombres en el $100 \%$, mientras que en el área de salud pública, las mujeres predominaron de igual forma en el 100\%. En cuanto al área clínica, se encontró que está conformada equitativamente, con el $50 \%$ de hombres y el $50 \%$ de mujeres (Figura 3).

En cuanto a las publicaciones editoriales, es importante mencionar que estos artículos son realizados por encargo del comité editorial. En el año 2013, por primera vez, se encargó el primer artículo editorial a una mujer (Figura 4).

\section{Discusión}

En la historia de la humanidad, la dicotomía hombre-mujer ha originado el establecimiento de estereotipos que han condicionado y limitado las potencialidades de uno u otro género en algunos ámbitos. Los esfuerzos mundiales para lograr una distribución igualitaria de los recursos y oportunidades entre hombres y mujeres han permitido reducir la brecha de género. Sin embargo, no se ha logrado la igualdad en todos los ámbitos, por lo que se observan diferencias a escala mundial ${ }^{1}$.

Hasta donde se conoce, es la primera vez que se evalúa la proporción por género en la producción de artículos del área médica en México. Se ha observado un evidente incremento en la participación de las mujeres en la autoría de artículos originales en el BMHIM. Por ello, la disminución en la brecha de género en la autoría podría ser un reflejo de la progresión de las mujeres en el área médica y de investigación ${ }^{20}$.

No cabe duda que, en general, la participación de las mujeres en la autoría de artículos científicos ha incrementado en el mundo. Sin embargo, la brecha de género persiste en la mayoría de los estudios, y no suele reportarse una superioridad en la proporción de mujeres en la autoría de artículos, como se identificó en las publicaciones del BMHIM en los últimos años.

Uno de los estudios más importantes en el que se evaluó la brecha de género en la autoría de revistas 


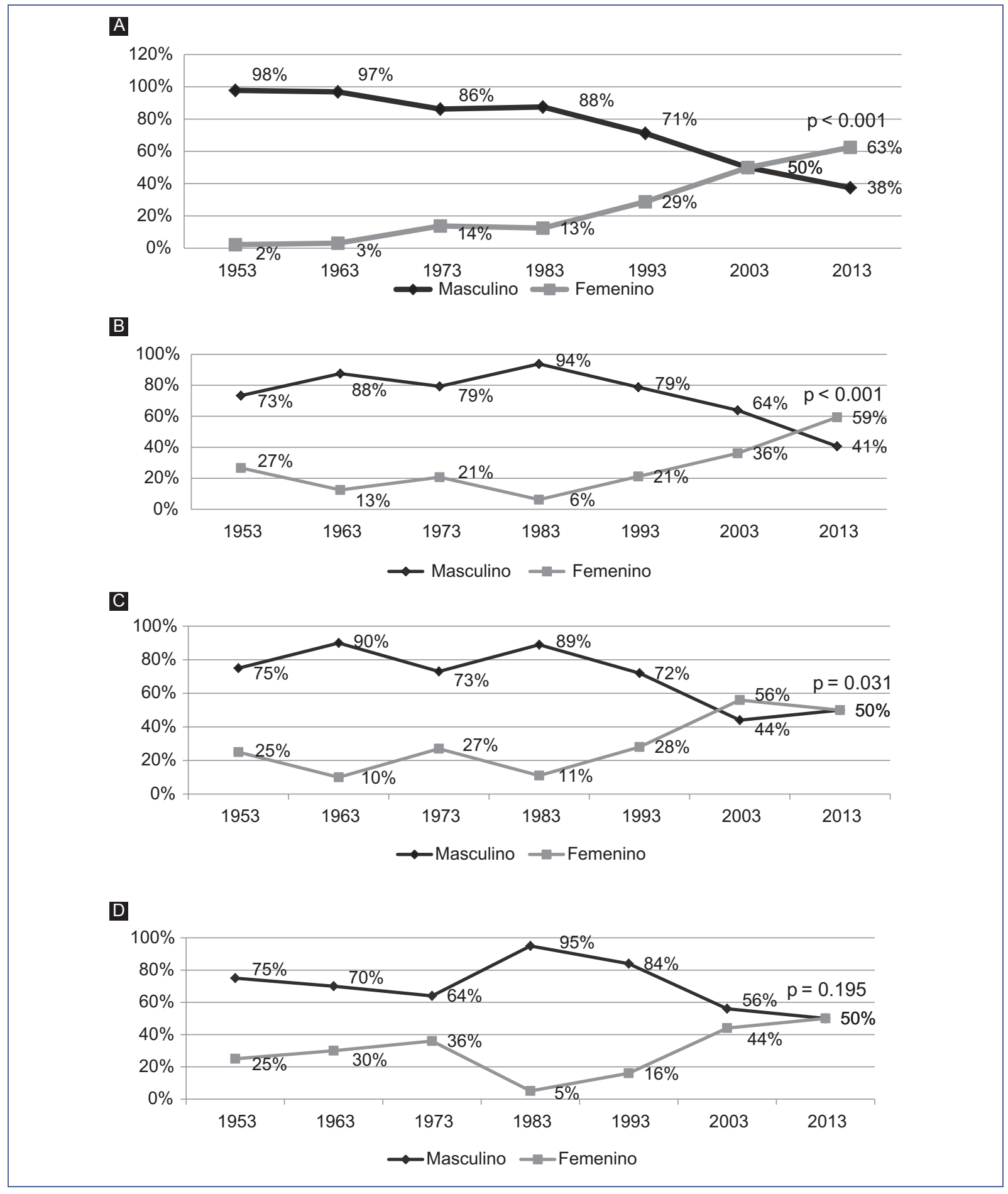

Figura 1. Tendencia de género como primer autor (A) y autor correspondiente (B), así como en estudios analíticos con respecto al primer autor (C) y el autor correspondiente (D) a través del tiempo en artículos publicados en el BMHIM.

científicas fue el publicado por Jagsi, et al. ${ }^{2}$ En este estudio, se analizaron seis revistas de alto impacto publicadas en los EE.UU. El estudio reportó un incremento de $5.9 \%$ en 1970 a $29.3 \%$ en 2004 ( $p<0.001)$ en la autoría de las mujeres como primera autora, y como autora principal o senior de 3.7 a $19.3 \%$ $(p<0.001)$. Un panorama similar fue reportado en Reino Unido ${ }^{12}$. 
Otros estudios también han reportado el incremento de la participación de las mujeres en la autoría de artículos, con cifras cercanas al $30 \%$ en las últimas décadas $3,6,7,7,10,15$, e incluso en el más reciente se menciona una cifra del $37 \% \%^{13}$. A pesar de ello, la mayoría de los estudios concluyen que la participación de las mujeres como autoras sigue siendo menor en comparación con los hombres, especialmente en lo referente a las áreas relacionadas con la cirugía $4,14,17$, lo cual ha sido relacionado con una menor participación de las mujeres en las especialidades quirúrgicas.

En el análisis específico de revistas del área pediátrica, el incremento en la participación de las mujeres como autoras ha sido mayor, y ha alcanzado cifras entre el 38.9 y el $53.2 \%$ como primeras autoras, y entre

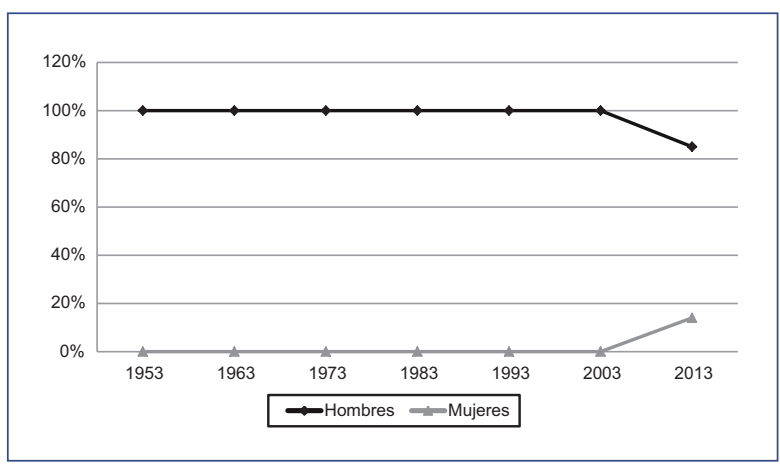

Figura 2. Conformación del comité editorial a través de los años. el 27.8 y el $38.0 \%$ como senior para la primera década del siglo $\left.X X\right|^{2,12}$. Si bien este porcentaje pareciera favorable, se debe considerar que la pediatría es una de las especialidades preferidas por las mujeres (dos a tres mujeres por cada hombre), por lo que se esperaría una mayor proporción de mujeres en la autoría dentro de esta especialidad ${ }^{21}$.

Si se considera que la proporción de mujeres en los programas de residencias médicas del Hospital Infantil de México Federico Gómez actualmente alcanza el $65.7 \%$ y la de investigadores/as de la institución el $50.5 \%$, la proporción de las autorías por parte de las mujeres estaría alcanzando una proporción similar $(\sim 60 \%)$. Es decir, estos resultados parecen acordes al incremento en la participación de las mujeres a la fuerza de trabajo del área pediátrica, lo que estaría reflejando una condición de equidad entre ambos géneros.

Aunque se han reportado casos en los que la producción científica de la mujeres excede la proporción de su participación en su área laboral ${ }^{17}$, existen otros ámbitos del área médica en la que mayor participación de mujeres no se ve reflejada en una mayor proporción de producción científica. Tal es el caso del campo de la enfermería, terreno tradicionalmente dominado por mujeres $(>90 \%)$, pero que, en términos de producción científica, su participación no es proporcional $(<70 \%)^{8}$.

Como se mencionó previamente, la mayoría de los estudios coinciden en que a pesar del incremento, la participación de las mujeres continúa siendo poca ${ }^{22}$.

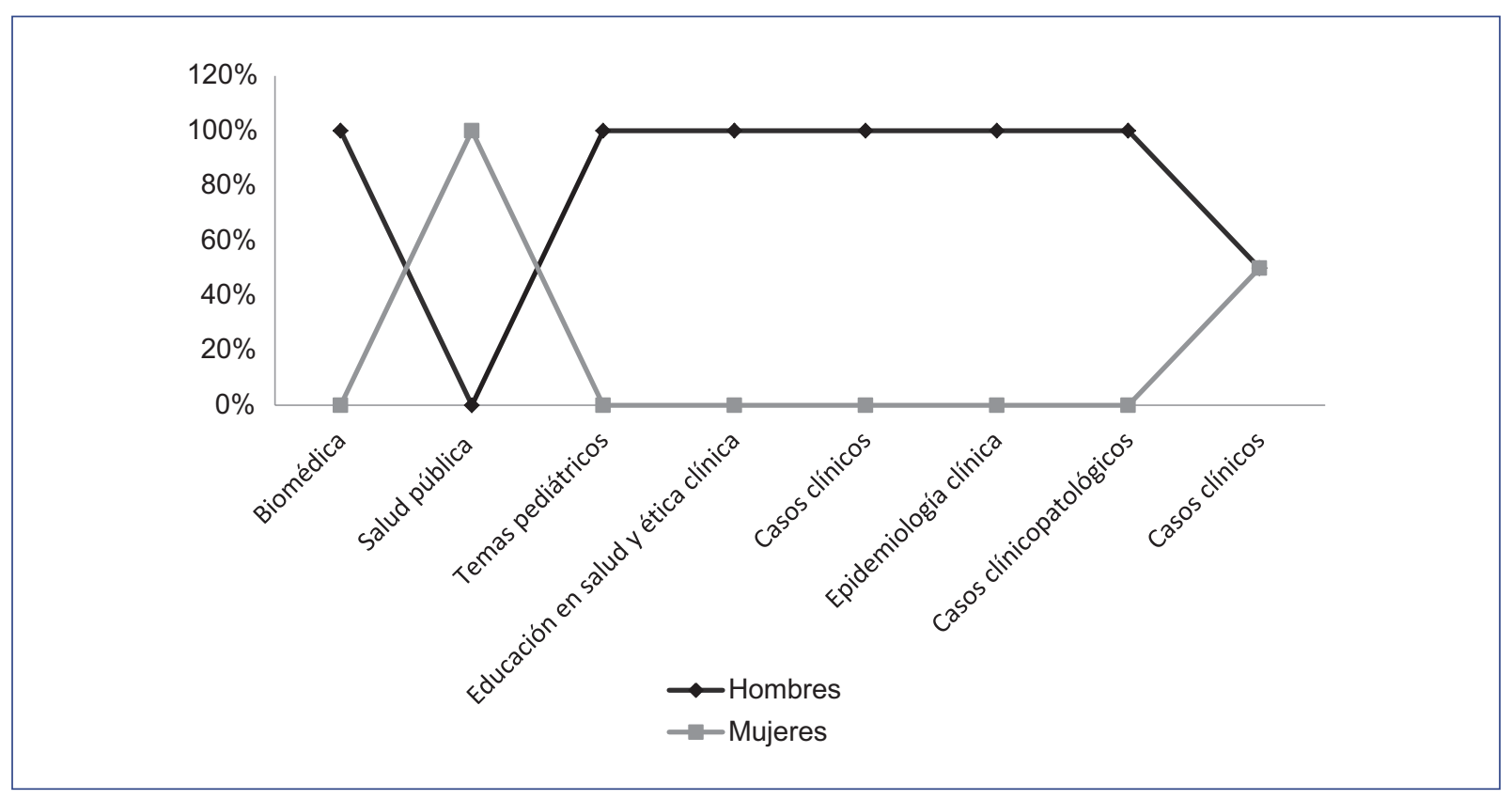

Figura 3. Conformación del comité editorial en el 2013 por área de especialidad. 


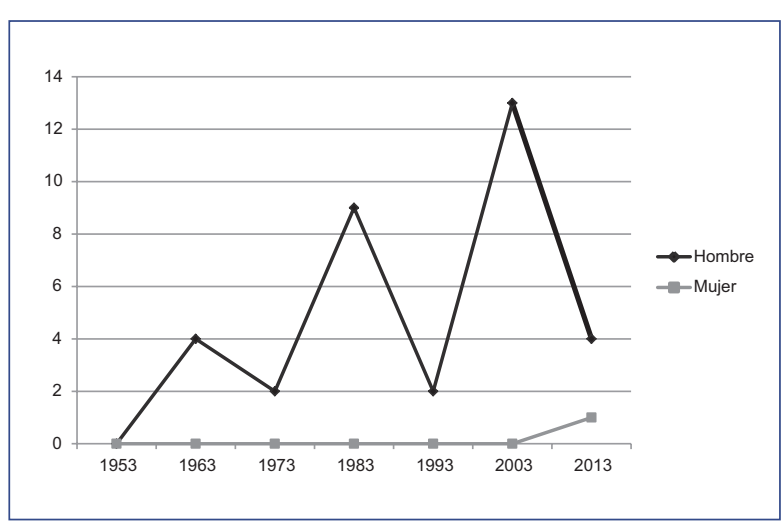

Figura 4. Publicaciones editoriales.

Dentro de las explicaciones de lo que se ha observado en el resto del mundo, se encuentran los temas relacionados con las diferencias en las metas laborales y de vida; es decir, el rol que deben desempeñar los hombres y las mujeres según el estereotipo de su género, determinado por la sociedad. Hace ver a la mujer como principal responsable de la crianza de los hijos y el hogar, y posiciona al hombre como proveedor, por lo que tiene más posibilidades de desarrollo laboral, coincidiendo con la hipótesis denominada "techo de cristal"19.

Se ha señalado que el éxito académico en el área médica requiere de una inversión importante de tiempo, que llega a ser incompatible con el cuidado de los hijos; las mujeres que son madres, al parecer publican menos y tienen menor apoyo de las instituciones en comparación a colegas varones con hijos ${ }^{23}$. Se ha señalado que, en general, el éxito académico en medicina requiere de 70 horas de trabajo por semana ${ }^{24}$, lo que puede resultar incompatible con los retos personales de vida.

Contrario a lo anterior, los presentes resultados señalaron una inversión en la relación hombre-mujer en la participación como primer autor y autor correspondiente. La explicación de estos resultados, en relación con los retos familiares y personales que dificultan el desarrollo académico de las mujeres en México, podría generar distintas hipótesis. Dentro de las posibles explicaciones se podrían proponer un incremento en el interés de las mujeres en las áreas médicas y de investigación, menor prioridad a los temas relacionados con la maternidad y la familia, mayor igualdad en las condiciones laborales, o bien una reducción en la discriminación por género. Todas estas situaciones han sido identificadas previamente como posibles barreras en la representación de las mujeres en la vida académica y científica ${ }^{23,25}$.

Por otra parte, una brecha de género en sentido opuesto, es decir, con una menor proporción en la producción científica por parte del género masculino, también contradeciría el principio de igualdad de género por el que se ha abogado en los últimos años. Analizando el otro lado de la moneda, la disminución en la proporción de la autoría de artículos científicos por parte de los hombres podría ser reflejo de un menor interés en los campos de la medicina y la investigación, lo que puede deberse, en parte, a que son carreras largas en las que la productividad empieza a una mayor edad y la remuneración económica puede no estar acorde al esfuerzo requerido para obtener un éxito académico.

A pesar de ello, se reconoce la limitante de que solamente se realizó el análisis de una pequeña parte del conocimiento en el área pediátrica que se genera en el país, y no se están evaluando otros medios de divulgación científica que podrían tener un mayor impacto académico o representar otras posiciones académicas con mayor liderazgo, y en las cuales se desconoce el balance de la participación hombre-mujer. Otra limitante del presente trabajo fue el enfoque en una sola revista, sin incluir todos los años de publicación, por lo que los resultados pueden no reflejar la totalidad de la investigación pediátrica en México ni mucho menos de otras especialidades. Adicionalmente, la baja representatividad de artículos con mayor evidencia científica publicados en el BMHIM (ensayos clínicos, metaanálisis) no permitió explorar, de forma detallada, la progresión de las mujeres en este tipo de estudios científicos. Por último, cuando el género de un autor no pudo ser definido en la valoración, se realizó una búsqueda exhaustiva por otros medios, lo cual podría no ser lo ideal para determinar el sexo de un autor.

En conclusión, en las últimas décadas ha existido un incremento significativo en la autoría de las mujeres en el BMHIM, que ha llegado incluso a una mayor proporción en relación al género masculino. Esto es un reflejo del papel actual de las mujeres en la medicina; en particular, en el área pediátrica. Si bien es cierto que se ha logrado un avance en el tema de equidad de género, aún hay trabajo que hacer respecto de los estereotipos que determinan los roles a lo femenino y lo masculino. Lo anterior es fundamental para considerar tanto a hombres como mujeres capaces de realizar las mismas funciones en el ámbito científico, laboral y personal.

\section{Responsabilidades éticas}

Protección de personas y animales. Los autores declaran que para esta investigación no se han realizado experimentos en seres humanos ni en animales.

Confidencialidad de los datos. Los autores declaran que han seguido los protocolos de su centro de trabajo sobre la publicación de datos de pacientes. 
Derecho a la privacidad y consentimiento informado. Los autores declaran que en este artículo no aparecen datos de pacientes.

\section{Financiamiento}

El presente estudio no requirió financiamiento.

\section{Conflicto de intereses}

Los autores declaran no tener ningún conflicto de intereses.

\section{Bibliografía}

1. The Global Gender Gap Report 2015. World Economic Forum (2015). Disponible en: http://reports.weforum.org/global-gender-gap-report-2016/.

2. Jagsi R, Guancial EA, Worobey CC, Henault LE, Chang Y, Starr R, et al. The "gender gap" in authorship of academic medical literature-a 35-year perspective. N Engl J Med. 2006;355(3):281-7.

3. Amering M, Schrank B, Sibitz I. The gender gap in high-impact psychiatry journals. Acad Med. 2011;86(8):946-52.

4. Bergeron JL, Wilken R, Miller ME, Shapiro NL, Bhattacharyya N. Measurable progress in female authorship in otolaryngology. Otolaryngol Head Neck Surg. 2012;147(1):40-3.

5. Dewan P, Gupta P, Choudhury P. Gender gap and Indian Academy of Pediatrics: still a long way to go! Indian Pediatr. 2007;44(8):598-600.

6. Özlem EA, Elçin ÖE, Ece U. Longitudinal analysis of female authorship of psychiatry articles in Turkey. Arch Neuropsychiatr. 2015;52:95-8.

7. Schrager S, Bouwkamp C, Mundt M. Gender and first authorship of papers in family medicine journals 2006-2008. Fam Med. 2011;43(3):155-9.

8. Shields L, Hall J, Mamun AA. The 'gender gap' in authorship in nursing literature. J R Soc Med. 2011;104(11):457-64.

9. Yun EJ, Yoon DY, Kim B, Moon JY, Yoon SJ, Hong SJ, et al. Closing the gender gap: increased female authorship in AJR and Radiology. AJR Am J Roentgenol. 2015;205(2):237-41.
10. Takayanagui OM, Livramento JA. The increasing female participation in authorship of articles published in neurology in Brazil. Arq Neuropsiquiatr. 2009;67(3b):914-6.

11. Shah DN, Huang J, Ying GS, Pietrobon R, O'Brien JM. Trends in female representation in published ophthalmology literature, 2000-2009. Digit J Ophthalmol. 2013;19(4):50-5.

12. Sidhu R, Rajashekhar $P$, Lavin VL, Parry J, Attwood J, Holdcroft A, et al. The gender imbalance in academic medicine: a study of female authorship in the United Kingdom. J R Soc Med. 2009;102(8):337-42.

13. Filardo G, da Graca B, Sass DM, Pollock BD, Smith EB, Martinez MA. Trends and comparison of female first authorship in high impact medical journals: observational study (1994-2014). BMJ. 2016;352:i847.

14. Kurichi JE, Kelz RR, Sonnad SS. Women authors of surgical research. Arch Surg. 2005;140(11):1074-7.

15. Feramisco JD, Leitenberger JJ, Redfern SI, Bian A, Xie XJ, Resneck JS, Jr. A gender gap in the dermatology literature? Cross-sectional analysis of manuscript authorship trends in dermatology journals during 3 decades. J Am Acad Dermatol. 2009;60(1):63-9.

16. Tinjum BE, Getto L, Tiedemann J, Marri M, Brodowy M, Bollinger M, et al. Female authorship in emergency medicine parallels women practicing academic emergency medicine. J Emerg Med. 2011;41(6):723-7.

17. Weiss DA, Kovshilovskaya B, Breyer BN. Gender trends of urology manuscript authors in the United States: a 35-year progression. J Urol. 2012;187(1):253-8.

18. Borrel C, Vives-Cases C, Domínguez-Berjón M, Álvarez-Dardet C. Las desigualdades de género en la ciencia: Gaceta Sanitaria da un paso adelante. Gac Sanit. 2015;29(3):161-3.

19. Colomer RC, Peiró PR. ¿Techos de cristal y escaleras resbaladizas? Desigualdades de género y estrategias de cambio en SESPAS. Gac Sanit. 2002;16(4):358-60.

20. Miller K. Policy and organizational implications of gender imbalance in the NHS. J Health Organ Manag. 2007;21(4-5):432-47.

21. Lambert EM, Holmboe ES. The relationship between specialty choice and gender of U.S. medical students, 1990-2003. Acad Med. 2005;80(9): 797-802.

22. S. Tornero $P$. Necesidad de la perspectiva de género en el anuario de Anales de Pediatría. An Pediatr. 2015; 83:225.

23. Carr PL, Ash AS, Friedman RH, Scaramucci A, Barnett RC, Szalacha L, et al. Relation of family responsibilities and gender to the productivity and career satisfaction of medical faculty. Ann Intern Med. 1998;129(7):532-8.

24. Hamel MB, Ingelfinger JR, Phimister E, Solomon CG. Women in academic medicine-progress and challenges. N Engl J Med. 2006;355(3):310-2.

25. Edmunds LD, Ovseiko PV, Shepperd S, Greenhalgh T, Frith P, Roberts NW, et al. Why do women choose or reject careers in academic medicine? A narrative review of empirical evidence. Lancet. 2016; 388(10062):2948-58 